\title{
Sex-Specific Neural Processing of Emotional Musical Stimuli in Depressed and Non-Depressed Individuals
}

\author{
Caroline Chan ${ }^{1}$, Noelle Almonte ${ }^{1}$, Chin $\mathrm{Chu}^{1}$, Zhishun Wang ${ }^{2^{*}}$ \\ ${ }^{1}$ River Dell High School, Oradell, New Jersey, USA \\ ${ }^{2}$ Department of Psychiatry, Columbia University and New York State Psychiatric Institute, New York, NY, USA
}

*Corresponding Author: Zhishun Wang, Department of Psychiatry, Columbia University and New York State Psychiatric Institute, New York, NY, USA, Email: zw2105@columbia.edu

\begin{abstract}
The present study investigated sex differences in functional responses to emotional musical stimuli in both depressed and non-depressed participants. Data were obtained from a dataset published by Rebecca J. Lepping et al. on Open fMRI, an open-access functional magnetic resonance imaging (fMRI) database (www.openfmri.org). Nineteen participants (8 males, 11 females) diagnosed with Major Depressive Disorder $(M D D)$ and 20 never-depressed (ND) participants (9 males, 11 females) were studied underthree different stimuli: emotionally neutral "pure tones", positive musical stimuli, and negative musical stimuli. Each participant underwent five sessions of fMRI scans each consisting of a 31.5-second musical sample followed by 31.5 seconds of pure tones, separated by a three-second response period, repeated four times per session. The analysis of the functional imaging data indicated that compared with never-depressed females, neverdepressed males had greater activations in primary auditory area and superior temporal gyrus, in response to both positive and negative music; while never-depressed females had greater activations than neverdepressed males in thalamus and amygdale in response to negative music. The analysis results also indicated that compared with depressed females, depressed males had greater activations in prefrontal cortex in response to positive music as well as greater activations in left palladium in response to negative music. These findings were not reported in the original study conducted by Rebecca J. Lepping et al.
\end{abstract}

Keywords: Depression, Sex-specific neural activity, Functional Magnetic Resonance Imaging, Brain musical processing.

\section{INTRODUCTION}

Music is a complex auditory emotion-inducing stimulus that is often used as a method of emotion regulation and manipulation [1]. Previous studies have demonstrated that neural processing of musical stimuli is a complex process involving prefrontal, temporal, and limbic structures, with pitch processing occurring in the primary auditory cortex (Heschl's gyrus) and higher-level melody extraction thought to occur outside of the primary auditory cortex in the superior temporal gyrus (STG) and planumpolare [16]. Other studies have observed increased activation of the amygdala, hippocampus, and temporal poles in response to unpleasant (negative valence) music [13]. Lesion studies of participants with amygdala damage and temporal lobe excisions including the amygdala indicate that the amygdala in particular is integral to the recognition and processing of scary and sad music $[8,9]$. Additional studies indicate the involvement of the inferior frontal gyrus (IFG) in recognition of incongruous chord sequences $[17,13]$ and the superior frontal gyrus (SFG) in working pitch memory [6]; nevertheless, many of these studies differ widely in stimulus selection and methodology, and the mechanisms by which the brain responds to and practices music are not fully understood.

Despite this, a growing body of evidence suggests that musical stimuli can generate positive, lasting effects on listeners. A study conducted by Castillo-Perez et. al observed depressive symptoms of patients with low- to medium-grade depression, and found fewer depressive symptoms among patients who received daily music therapy using classical and baroque instrumental music than among patients given weekly conductive-behavioral-based psychotherapy [5]. Castillo-Perez's group also observed an increase in interest of depressed participants undergoing music therapy in the presented music despite the frequent failure to 
find interest in positive stimuli common to depressed patients [5]. In response to the observation that depression is caused by decreased levels of dopamine [15], Sutoo and Akiyama found that musical stimuli increased dopamine levels in murine models, and hypothesized that music can be used as a tool to relieve symptoms associated with diseases involving dopamine dysfunction, such as depression [18]. A PET study conducted by Blood and Zatorre also observed that when particular selections of music created an intensely pleasureable response of "chills" in participants, changes in cerebral blood flow occurred in regions associated with reward and emotion, representing the potential for music as a personalized therapy tool to induce positive emotion [3]. Other studies have noted possible short-term positive effects of musical stimuli on spatial-temporal reasoning (the so-called "Mozart Effect") in adults and projected longterm enhanced spatial-temporal reasoning in children who listened to Mozart's music and received basic musical training [11]. As such, investigating neural responses to musical stimuli constitutes an important research initiative.

Numerous studies have investigated sex differences in cognitive $[2,19,10,7]$ and emotional responses $[4,12]$ to various verbal, auditory, and visual stimuli, often with mixed results. However, despite the emerging prominence of music as a therapy tool, few have examined gender differences in response to musical stimuli. Although a study conducted by Miles et al., 2016 observed a female advantage at recognizing familiar melodies and an EEG study conducted by Koelsch et al., 2003 reported early negativity in response to musical stimuli generated bilaterally in adult females and unilaterally dominant in the right hemisphere in adult males, to our knowledge no previous study has examined sex differences in response to musical stimuli through methods with higher spatial resolution $[14,13]$.

Using functional magnetic resonance imaging (fMRI), this study investigated gender differences in response to emotional musical stimuli in never-depressed and depressed participants. We reanalyzed public-access data acquired by Rebecca Lepping et al in a 2016 study investigating differences in processing of positively and negatively valenced musical and nonmusical stimuli between depressed and never-depressed (ND) individuals [1]. Lepping's group observed differing patterns of activation in the anterior cingulate cortex (ACC) depending on the stimulus type (musical or nonmusical) and valence, but did not examine sex differences in these responses [1]. As such, this study reanalyzed their data, with respect to musical stimuli only, to determine what, if any, sex differences exist in responses to music.

\section{MATERials AND METHOdS}

\subsection{Data Acquisition}

Data was acquired from Open fMRI (https://openfmri.org/), an online public-access neuroimaging database. The dataset, entitled "Neural Processing of Emotional Musical and Nonmusical Stimuli in Depression" (accession number ds000171) was originally published by Lepping et al. on May 26, 2016.

\subsection{Participants}

This study tested twenty ND participants, 9 males and 11 females (Males: $n=9$, Range $e_{\text {MALE }}$ AGE $=18-52$; Females: $n=11$, Range FEMALE AGE $=18-59)$ with no prior history of depression or of other psychiatric disorders and nineteen participants with MDD, 9 males and 10 females (Males: $n=9$, Range MALE AGE $=19-$ 56; Females: $n=10$, Range Female AGE $=18-$ 52 ), as determined by the administered nonpatient Structured Clinical Interview for DSM disorders [1]. All MDD participants were currently experiencing a depressive episode and were not taking medication for depression during the time of scanning, had no prior or current manic episodes or alcohol abuse, and had no comorbid anxiety disorders [1]. All participants were right-handed with no contraindications for MRI or conditions or medication affecting blood flow or brain function [1]. Participants in both groups did not significantly differ in age, length of education, or length of musical training [1].

\subsection{Stimuli}

Samples of positive and negative emotioninducing instrumental Western art music were selected and validated by Lepping et al. from the International Affective Digital Sound set (IADS) [1]. The final stimuli set used by Lepping et al. in this study included 36 musical (18 negative valence, 18 positive valence) samples, as well as 9 pure tone samples that were validated as emotionally neutral in a prior study conducted by the same group and used as a baseline for comparison [1]. The samples differed only in valence and did not differ in arousal ratings [1]. 


\subsection{Imaging Parameters}

Scans were obtained by Lepping's group using a 3 Tesla Siemens Skyra scanner [1]. Lepping's group immobilized participants' heads at a slice acquisition angle of $40^{\circ}$ using cushions, and obtained high-resolution T1-weighted anatomical images $(\mathrm{TR} / \mathrm{TE}=2300 / 2.01 \mathrm{msec}$, $\mathrm{FOV}=256 \mathrm{~mm}$, slice thickness $=1 \mathrm{~mm}$, matrix $=256 \times 192$, flip angle $=9^{\circ}$ ) $[1]$. Lepping's group conducted five functional scanning runs, each acquired in 50 oblique interleaved axial slices with a thickness of $3 \mathrm{~mm}$ (TR/TE = $3000 / 25 \mathrm{msec}$, FOV $=220 \mathrm{~mm}, 105$ data points, flip angle $\left.=90^{\circ}\right)$ [1].

\subsection{Task Paradigm}

A single scanning session was conducted by Lepping et al. with five functional scanning runs and anatomical scanning [1, Figure 1]. Functional runs included alternating blocks of auditory stimuli (Duration $=31.5$ seconds), each block containing three music samples of the same valence and a three-second response period, and blocks of baseline emotionally neutral pure tones; consecutive blocks of auditory stimuli alternated between positive and negative valence [1]. To prevent interference of the MR scanner's noise with the auditory stimuli, Lepping's group presented musical samples through MR compatible earbuds at volume levels previously adjusted before scanning occurred and covered the earbuds with noise-cancelling headphones to reduce scanner noise [1]. During the three-second response periods, participants were instructed to rate the preceding music block as positive or negative in valence to ensure they had been paying attention to the stimuli [1]. Following scanning sessions and outside of the scanning environment, Lepping's group instructed participants to rate stimuli for arousal and valence based on how each sample made them feel, and recorded responses via mouse click [1].

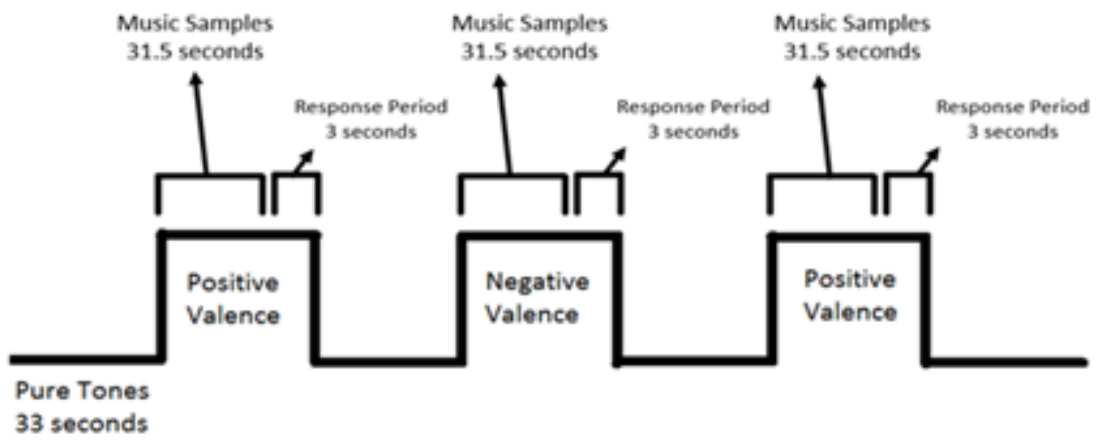

Figure1. Experimental Paradigm

The Experimental paradigm used by Lepping et al., including alternating blocks of positive and Negative music separated by blocks of baseline pure tones (1)

\subsection{Data Analyses}

The analyses of image data included two steps: image preprocessing and statistical analyses:

- fmri Preprocessing: fMRI data were analyzed using (http://www.fil.ion.ucl.ac.uk/spm/), running on MATLAB 2016b. Images were temporally aligned using the $25^{\text {th }}$ slice as a reference $(\mathrm{TR}=3.0 \mathrm{~s}$, interleaved slices $)$, spatially realigned, normalized using the realigned mean image, and smoothed using dependency on the temporal alignment module.

- fMRI Statistical Analysis: Preprocessed data was analyzed at the first level (single session) using the general linear model with three conditions: positive music, negative music, and response period, as specified by Lepping's group. Onsets, durations, and conditions were specified using SPM's Specify $1^{\text {st }}$-Level and Estimate features. Three contrasts were created per participant at the first level: effect of positive music only, effect of negative music only, and comparison between positive and negative music (analogous contrasts were also created for nonmusic runs, but were excluded from final results, as the objective of this study was to examine sex differences in response to musical stimuli only). Data for music runs was then analyzed at the second level using one-sample t-tests within male and female participant groups (contrasts: Male>Female, abbreviated $M v s F$, and Female>Male, abbreviated FvsM, for both normal control and 
depressed participants) and two-sample Ttests between male and female participant groups (also with separate t-tests for normal control and depressed participants).

\section{Results}

We used SPM Anatomy Toolbox (http://www.fil.ion.ucl.ac.uk/spm/) to overlay functional image results onto MNI template for visualization and to obtain the activation coordinates. We summarized results as follows.

\subsection{Never-Depressed Participants}

- Positive Music: The second-level analysis of responses to positive musical stimuli in never-depressed participants showed greater activation in never-depressed males than never-depressed females $(\mathrm{M}>\mathrm{F})$ in the right superior temporal gyrus $\quad(p<0.001$, uncorrected, $k=0$ ) [Figure 2]. Males also showed greater activation in prefrontal areas (right superior frontal gyrus, left inferior frontal gyrus, $p<0.001$, uncorrected, $k=0$ ). Never-depressed females were not observed to have any significantly greater activation than never-depressed males in response to positive music.

\section{In Response to Positive Music}

Control, Male > Female
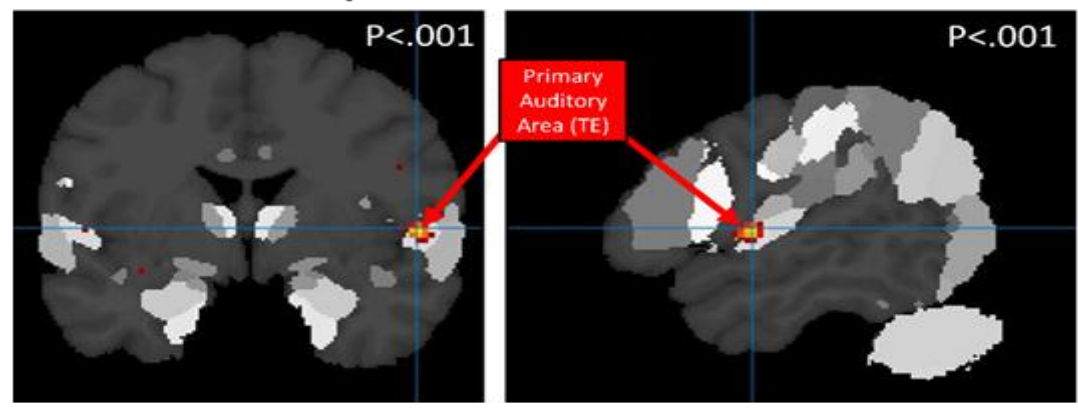

Figure2

- Negative Music: In response to negative music, never-depressed males were also found to have a higher activation than never-depressed females $(\mathrm{M}>\mathrm{F})$ in the right superior temporal $\operatorname{gyrus}(p<0.001$, uncorrected, $k=0)$ [Figure 3] and in left inferior frontal gyrus $\quad(p<0.025$,

Control, Male > Female In Response to Negative Music

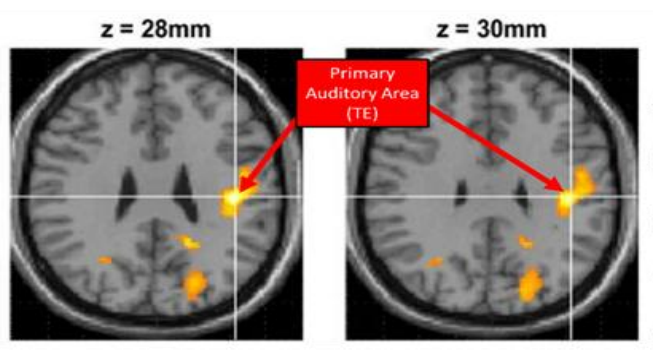

$\mathrm{P}<.025$, extent threshold $=30$ voxels

Figure3

\subsection{MDD Participants}

- Positive Music: In response to positive music, depressed males showed greater activation than depressed females $(\mathrm{M}>\mathrm{F})$ in the prefrontal cortex (left superior frontal gyrus, right inferior frontal gyrus, $p<0.001$ uncorrected, $k=0$ ). Depressed males also showed greater activation than depressed uncorrected, $\quad k=30$ ). $\quad$ Never-depressed females were found to have a higher activation than never-depressed males $(\mathrm{F}>\mathrm{M})$ in the thalamus and the left amygdala $(p<0.001, \quad$ uncorrected, $k=0)$ [Figure 4].
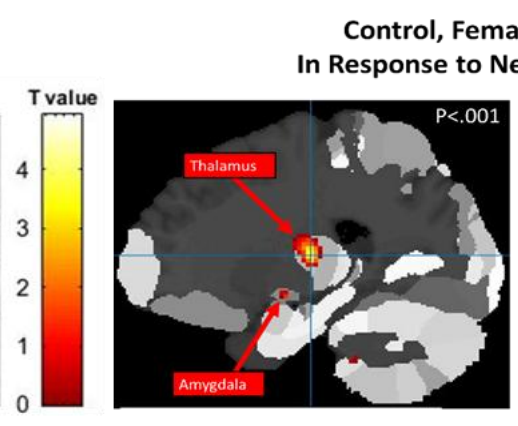

Figure4

males in auditory areas (left superior frontal gyrus, $p<0.025$, uncorrected, $k=30$ ) as well as in other locations in the temporal lobe (right middle temporal gyrus, $p<0.025$, Uncorrected, $k=30$ ) [Figure 5]. Depressed females were not observed to have any significantly greater activation than depressed males in response to positive music. 


\section{Depression \\ Male > Female \\ In Response to \\ Negative Music}

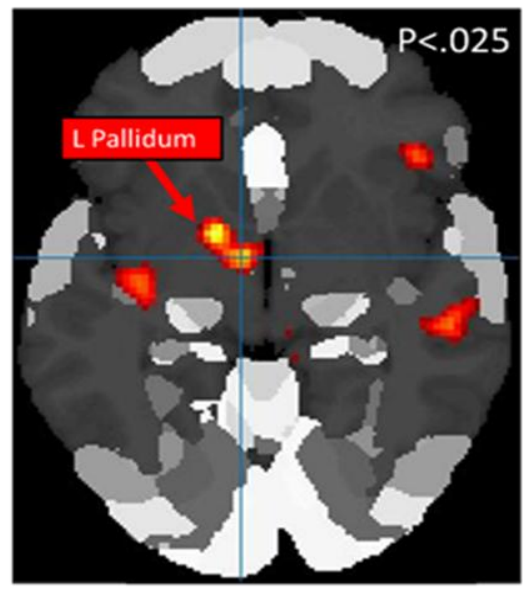

Extent Threshold $=30$ voxels
Depression

Male > Female

In Response to

Positive Music

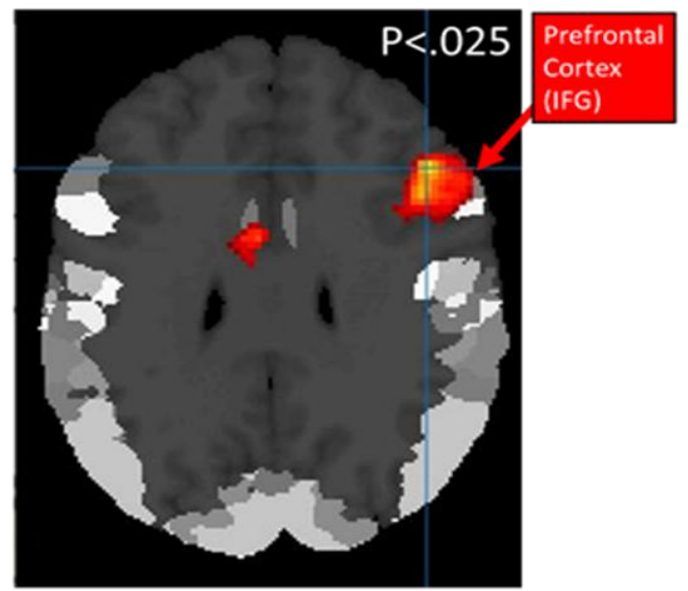

Extent Threshold $=30$ voxels

Figure 5

- Negative Music: In response to negative music, depressed males showed greater activation than depressed females $(\mathrm{M}>\mathrm{F})$ in the left pallidum and the left inferior temporal gyrus $(p<0.001$, uncorrected, $k=0$ ) [Figure 5]. As in response to positive music, depressed males also displayed greater activation than depressed males in temporal lobe areas (left inferior and superior temporal gyri, $\quad p<0.025$, uncorrected, $k=30$ ). Depressed females were not observed to display any significantly greater activation than depressed males in response to negative music.

\section{DISCUSSION}

We have reanalyzed the fMRI data that we downloaded from an open fMRI dataset resource, Open fMRI (https://openfmri.org/), in a study performed by Rebecca Lepping et al [1]. The functional image data were acquired from 19 participants (8 males, 11 females) diagnosed with major depressive disorder (MDD) and 20 age- and sex-matched controls (never-depressed, 9 males, 11 females) while the performance of an emotional musical stimulation task. The original aim of that study was to investigate differences of neural activations under positive and negative musical stimulations between MDD individuals and never-depressed controls. The aim of our study was to use the same dataset to investigate gender differences of neural activations in response to positive and negative musical stimulations. We have found that compared with never-depressed females, never-depressed males had greater activations in primary auditory area and superior temporal gyrus, in response to both positive and negative music; while never-depressed females had greater activations than never-depressed males in thalamus and amygdala in response to negative music. Our results also indicated that compared with depressed females, depressed males had greater activations in prefrontal cortex in response to positive music as well as greater activations in left pallidum in response to negative music. Our findings were not reported in the original study of this dataset [1].

\section{REFERENCES}

[1] Lepping RJ, Atchley RA, Chrysikou E, Martin LE, Clair AA, Ingram RE, et al. (2016) Neural Processing of Emotional Musical and Nonmusical Stimuli in Depression. PLoS ONE 11(6): e0156859.doi:10.1371/journal. Pone .0156859

[2] Wang ZS, Jacobs RH, Marsh R, Horga G, Qioa JP, Warner V, et al. Sex-specific neural activity when resolving cognitive interference in individuals with or without prior internalizing disorders. Psychiatry Research-Neuroimaging. 2016; 249:76-83.

[3] Blood AJ, Zatorre RJ. Intensely pleasurable responses to music correlate with activity in brain regions implicated in reward and emotion. Proceedings of the National Academy of 
Sciences of the United States of America. 2001; 98(20):11818-11823. doi:10.1073/pnas. 191355 898.

[4] Bradley M. M., Codispoti M., Sabatinelli D., Lang P. J. (2001). Emotion and motivation II: sex differences in picture processing. Emotion 1 300-319. 10.1037/1528-3542.1.3.300

[5] Castillo-Perez S., Gomez-Perez V., Calvillo Velasco M., Perez-Campos E., Mayoral M. (2010). Effects of music therapy on depression compared with psychotherapy. The Arts in Psychotherapy, 37(5), 387-390. doi:10.1016/ j.aip.2010.07.001

[6] Gaab N., Gaser C., Zähle T., Jäncke L. \&Schlaug G. Functional anatomy of pitch memory - an fMRI study with sparse temporal sampling. Neuroimage 19, 1417-1426, doi: 10.1016/s1053-8119(03)00224-6 (2003).

[7] Goldstein, J. M., Poldrack, R., Breiter, H.C., Makris, N., Goodman, J. M., Jerram, M., ...Seidman, L. J. (2005). Sex differences in prefrontal cortical brain activity during fMRI of auditory verbal working memory. Neuro psychology, 19(4), 509-519. DOI: 10.1037/ 0894-4105.19.4.509

[8] Gosselin N., Peretz I., Johnsen E., Adolphs R. (2007). Amygdala damage impairs emotion recognition from music. Neuropsychologia 45, 236-244. 10.1016/j. neuropsychologia. 2006. 07. 012

[9] Gosselin N. et al. Impaired recognition of scary music following unilateral temporal lobe excision. Brain 128, 628-640 (2005).

[10] Hugdahl K, Thomsen T, Ersland L. Sex differences in visuo-spatial processing: an fMRI study of mental rotation. Neuropsychologia. 2006; 44:1575-83. DOI: 10.1016/j.neuropsychologia. 2006. 01.026

[11] Jenkins JS. The Mozart effect. Journal of the Royal Society of Medicine. 2001; 94(4):170172.
[12] Kelly MM, Tyrka AR, Anderson GM, Price LH, Carpenter LL. Sex differences in emotional and physiological response to the Triers Social Stress Test. Journal of Behavioral Theory and Experimental Psychiatry. 2008; 39: 87-98.

[13] Koelsch S, Maess B, Grossmann T, Friederici AD. Electric brain responses reveal gender differences in music processing. Neuroreport. 2003; 14(5). DOI: 10.1097/01.wnr.00000 65762. 60383.67

[14] Miles SA, Miranda RA, Ullman MT. Sex Differences in Music: A Female Advantage at Recognizing Familiar Melodies. Frontiers in Psychology. 2016; 7:278. Doi: 10.3389/fpsyg. 2016. 00278 .

[15] Nestler E. J. et al. . Neurobiology of depression. Neuron 34, 13-25 (2002).

[16] Patterson R. D., Uppenkamp S., Johnsrude I. S., Griffiths T. D. (2002). The processing of temporal pitch and melody information in auditory cortex. Neuron 36, 767-776. 10.1016/ s0896-6273(02)01060-7

[17] Royal I, Vuvan DT, Zendel BR, Robitaille N, Schönwiesner M, Peretz I. Activation in the Right Inferior Parietal Lobule Reflects the Representation of Musical Structure beyond Simple Pitch Discrimination. Snyder J, ed. PLoS ONE. 2016; 11(5): e0155291. doi:10. 1371/journal. pone. 0155291 .

[18] Sutoo D., Akiyama K. (2004). Music improves dopaminergic neurotransmission: demonstration based on the effect of music on blood pressure regulation. Brain Res. 1016 255-262. 10.1016/j.brainres.2004.05.018

[19] Wallentin M. (2009). Putative sex differences in verbal abilities and language cortex: a critical review. Brain Lang. 108 175-183. 10.1016/ j.bandl.2008.07.001

Citation: Caroline Chan, Noelle Almonte, Chin Chu, Zhishun Wang2. Sex-Specific Neural Processing of Emotional Musical Stimuli in Depressed and Non-Depressed Individuals. ARC Journal of Neuroscience. 2018; 3(1):26-31. doi:dx.doi.org/10.20431/2456-057X.0301005.

Copyright: (C) 2018 Authors. This is an open-access article distributed under the terms of the Creative Commons Attribution License, which permits unrestricted use, distribution, and reproduction in any medium, provided the original author and source are credited. 